\title{
Cross Loading Motor Force Control System Design and Realization
}

\author{
Jinhong $\mathrm{LI}^{1, \text { a }}$, Shutao Zheng ${ }^{2, \mathrm{~b}}$ \\ ${ }^{1}$ Harbin Cambridge University, Harbin, China \\ ${ }^{2}$ School of Mechanism Engineering Harbin Institute of Technology, Harbin, China \\ alijinhong80@163.com, bzhengshutao77@163.com
}

Keywords: Cross Loading, Force control, Frequency characteristic, Motor, RCP

\begin{abstract}
Cross loading equipment is one of the important test equipments in airplane, aviation and shipping field. It is used to test the performance of rudder used in airplane, ship and missile etc in laboratory. A cross loading equipment based torque motor was processed. This paper gives the design process which includes mechanical hardware design, control system design and software development. In order to shorten development cycle and reduce program error used $\mathrm{C} / \mathrm{C}++, \mathrm{RCP}$ technology based Matlab/Simulink was used. Experiments shows us the cross loading system can realize the designed performance.
\end{abstract}

\section{Introduction}

Rudder loading simulation system is the important equipment which was used in industry, university and research institution to act force to rudder to test and verify the performance. Load simulation has two methods: hydraulic-driven and electric-driven. Hydraulic-driven simulation system was always used in the large torque and high dynamic response field with the disadvantage of design complex, large volume and large inertia, Electric-driven simulation system was always used in lower torque and low dynamic response field ${ }^{[1-2]}$. According to the need of project, a kind of rudder motor load simulation system is designed based on high-powered single chip microcomputer and moment motor ${ }^{[3]}$. Math model of electric load and surplus torque influence factor were given, position forecast control strategy was present to solve surplus torque ${ }^{[4]}$. This paper shows you the whole development of cross loading simulator including mechanical design, control system design and software design. In order to reduce program error with traditional program language such as $\mathrm{C} / \mathrm{C}++$ and shorten the development cycle, Rapid Control Prototyping (RCP) technology based Matlab/Simulink was used. Part experiments have been finished which shows the high performance.

\section{Mechanical Design}

Mechanical structure is the important component in cross loading equipment. Fig. 1 is the components of cross loading equipment. Loading assembly are composed of a torque motor which was used to act torque, erection support which was used to fix motor and shaft coupling which was used to connect rudder and torque motor. Transition assembly was fixed on the lampstand which can move forward or back along the moving track through turn hand wheel clockwise or anticlockwise to suit the different size rudder. Base assembly have two functions, one was used to install fixture which can fasten the rudder to the requirement position to test, the other it provides a platform which person can adjust the rudder position easily and change the parts inside rudder.

All the mechanical structure was designed by CAXA software, which can process interface between parts directly. In the design process, according to the requirement, all kinds of form can be design. When the interface appears, designer can adjust the position and size easily to solve the problem. The other, the design result can shows us "what you see is what you get" which will help the consumer to judge the design whether satisfy the requirement. 


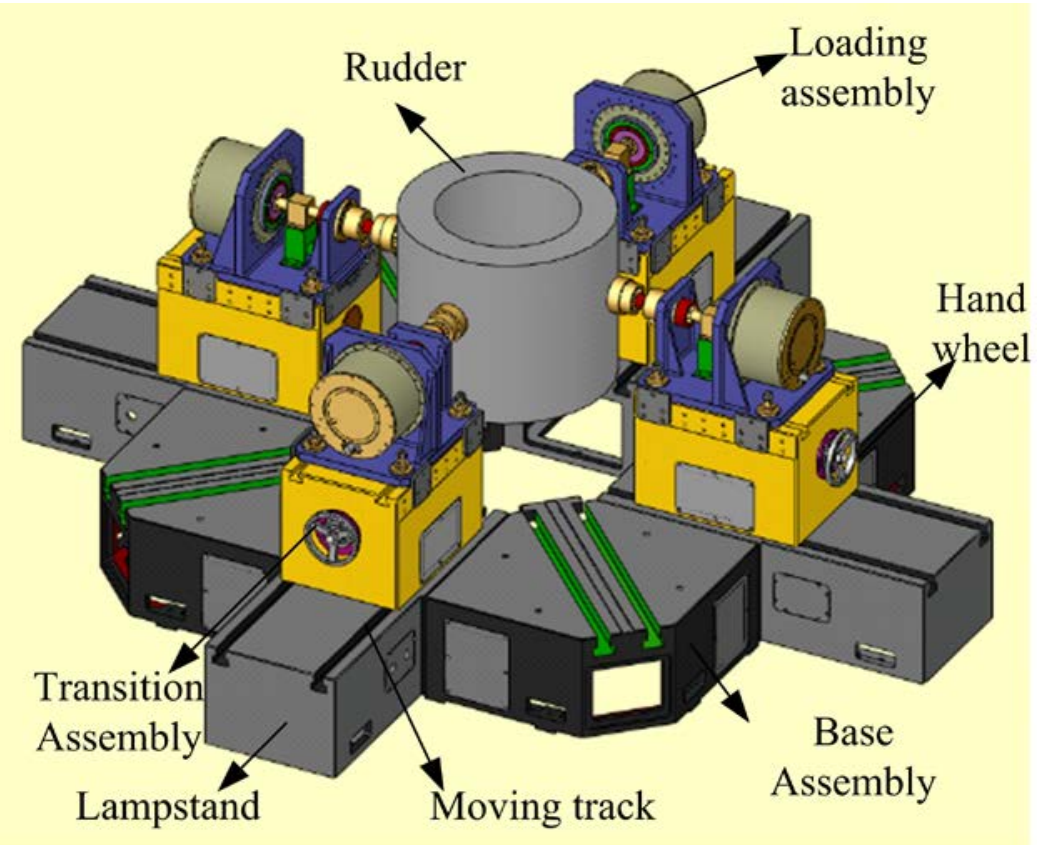

Fig.1 Mechanical Components of Cross Loading

\section{Control System Design}

Control system is the core of the cross loading simulator, it is composed of control system hardware and control software. With the rapid development of computer technology, digital control technology was used in control system widely. Considering the control precision and real-time performance, host-target communication method was used, host based PC was used to set control instruct and monitor the status of cross loading simulator, Target based X86 have two functions, one is used to realize the real-time data acquisition including $A / D, D / A$ and $I / O$, the other is to finish the real-time resolving of control system model. Fig. 2 is the hardware components of the control system.

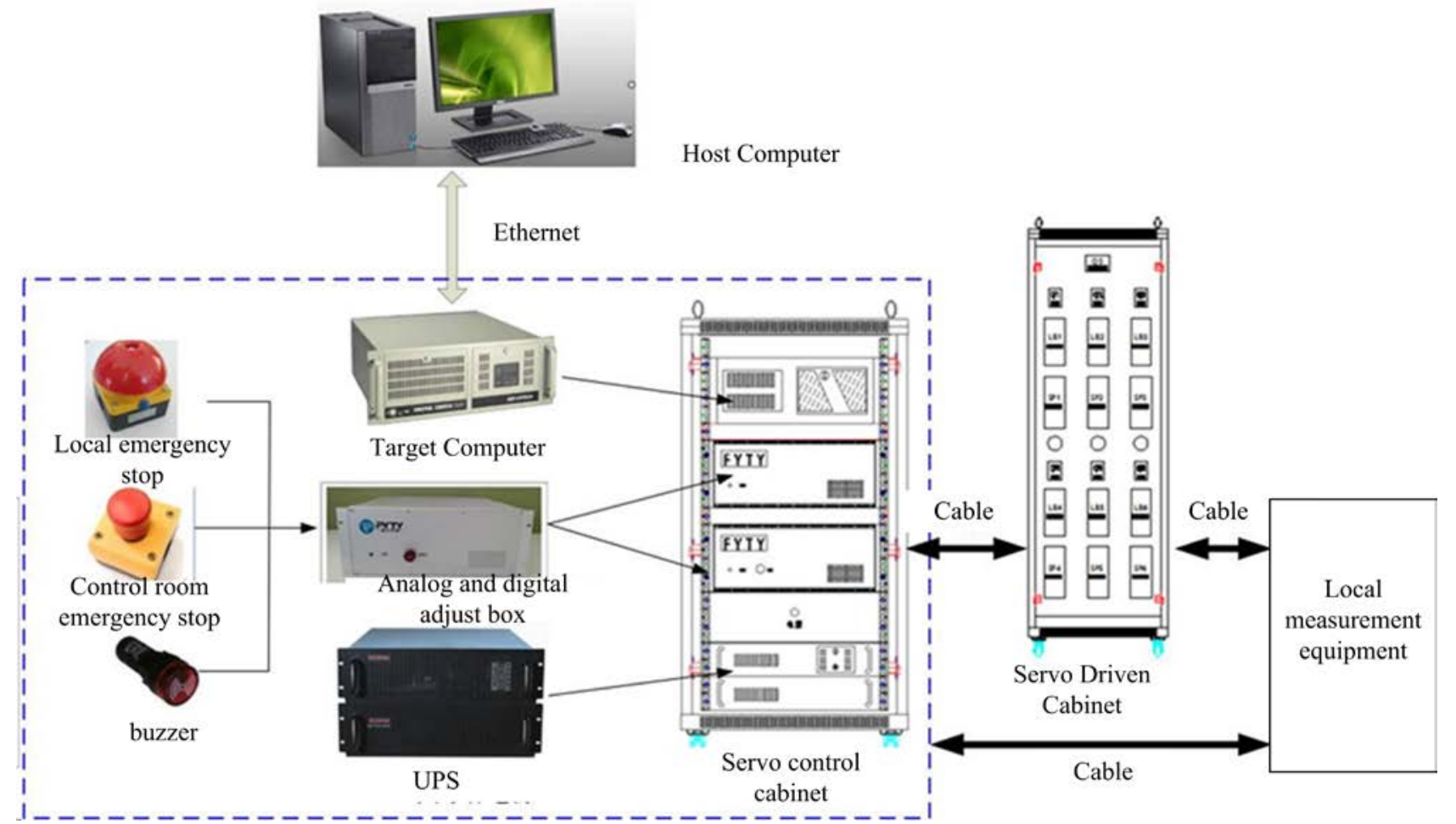

Fig.2 Hardware components of control system

Host computer is the computer with XP operation system which provides a friendly human-machine interactive interface with Labview ${ }^{[5]}$ GUI program language. Target computer is X86 computer based XPC-target with Real-time kernel. Ethernet is the main interactive method 
between host and target. In order to deal with the emergency fault, two emergency stop buttons were provided in control room and local. In order to point out the operator the fault message, a buzzer was fixed in servo control cabinet which was placed in the control room.

In order to reduce program error and shorten development cycle, Rapid Control Prototyping technology was used. Matalb/Simulink ${ }^{[6]}$ is used to develop the control model of cross load simulator, Fig.3 shows you the development model. It includes signal enable block which was used to control motor run or stop, watchdog check block which was used to check whether or not computer system halted, data acquisition block which was used to gain all kinds of signals of local measurement equipment adopting PCL-816, PCL-818HD, ACL-6162 and PCL-731, controller block which was use to realize the torque loop control with PID control and communication block using UDP protocol to change information between host and target. Auto real-time code generation technology was used to gain the real-time running program with the sample time $1 \mathrm{~ms}$.

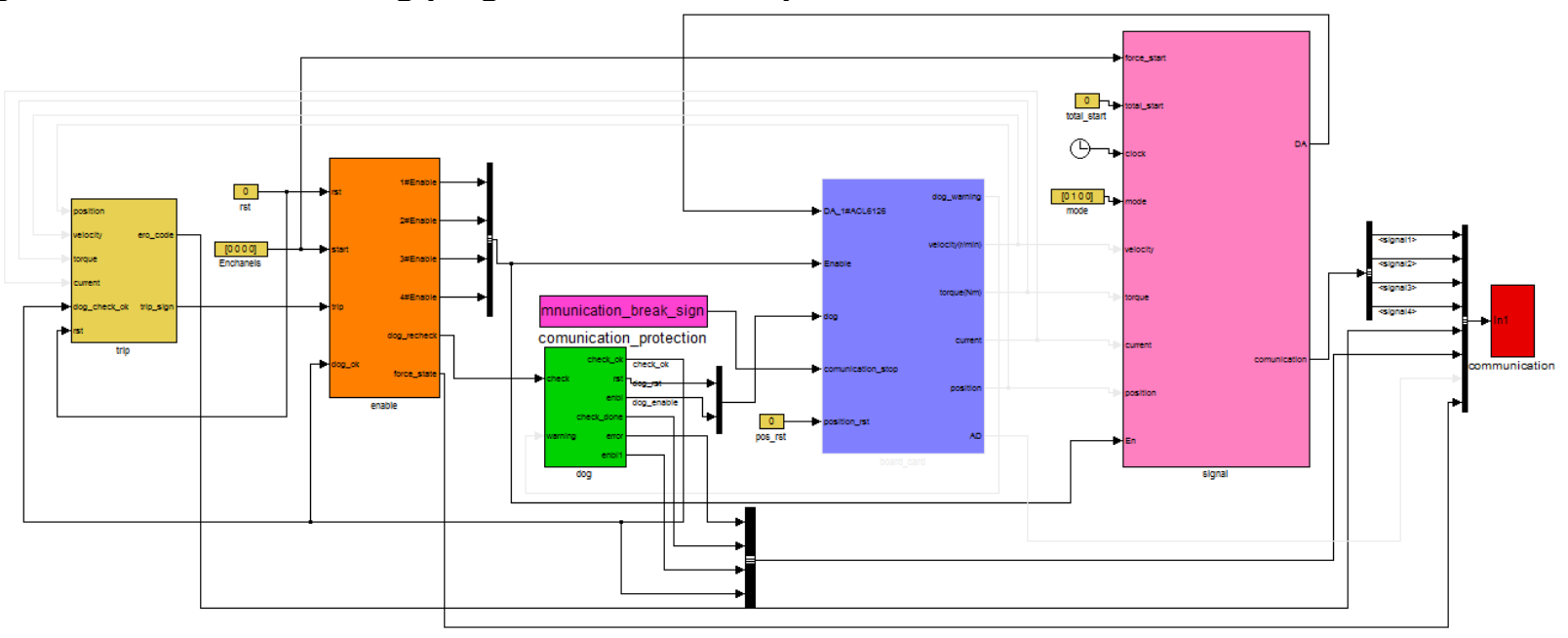

Fig.3 Control model based Matlab/Simulink

\section{Experiments}

According to the control model, part equipments have been processed. Frequency character of cross loading system was first given. Fig. 4 is the frequency character of closed loop with different gain. Fig.5 is the torque curve between reference signal and feedback signal. The signal is sin wave $\mathrm{T}=220 * \sin \left(2 * \mathrm{pi}^{*} 10 \mathrm{t}\right)$. From the Fig. 5 we can conclude that the amplitude rate is $95.4 \%$ and phase error is $7^{\circ}$.

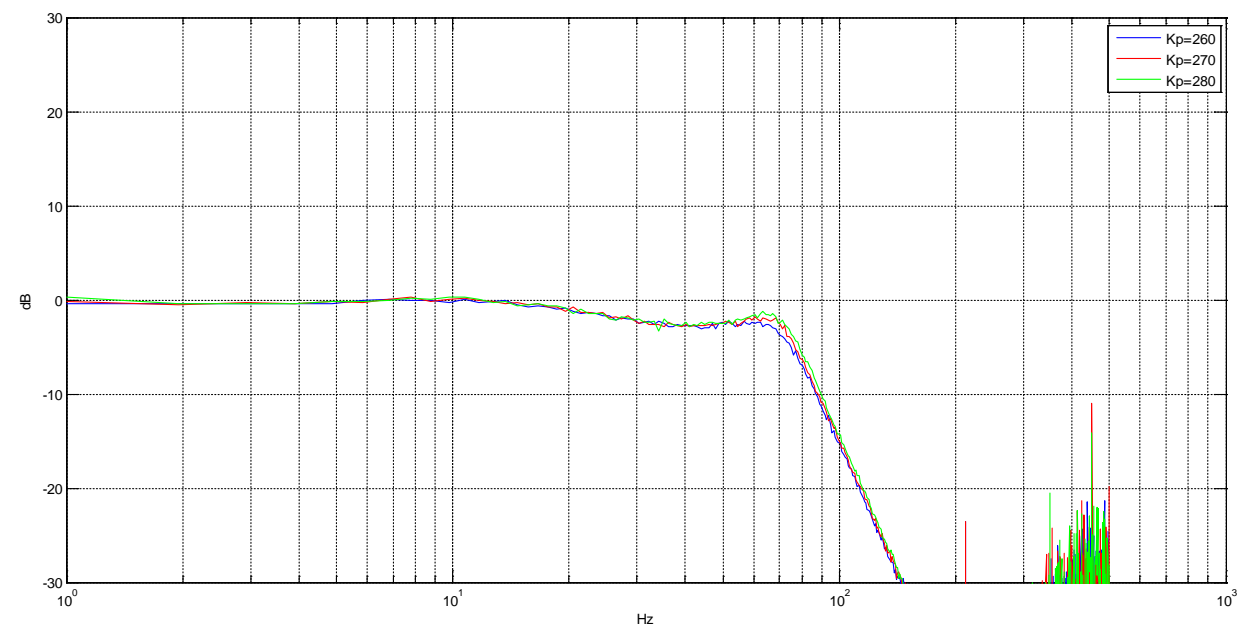

Fig.4 Velocity Loop Frequency Character with different gain 


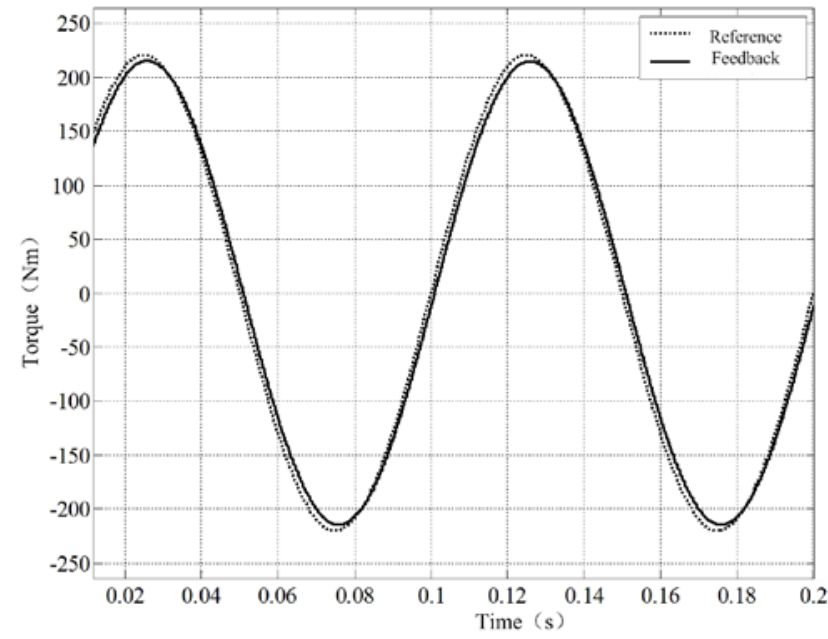

Fig.5 Torque curve

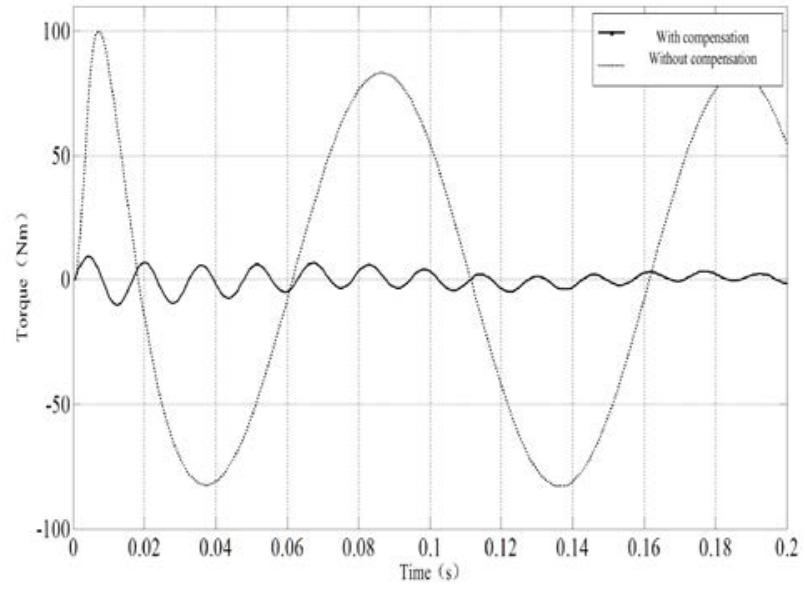

Fig.6 Surplus torque curve with or not compensation

\section{Summary}

Rudder loading simulation system is the important equipment which was used in industry, university and research institution. According to the test requirement, a cross loading simulator was designed. This paper shows the whole development process including mechanical structure, control system and software. In order to shorten development cycle and reduce program error, RCP technology was used, Part experiments shows that the designed cross loading simulator can fulfill the design requirement. Further deep research was processed.

\section{References}

[1] Zou Haifeng, Research on aircraft rudder motor servo loading system[J], Journal of system simulation ,2004(04).

[2] Li Yunhua, Surplus torque restraining method research of motor load simulator[J], Machine Tool \& Hydraulics, 1999(2).

[3] Chaohui Yuan, Tao Sun, Research on compound control method of rudder motor loading simulation system [J]. Computer measurement \&control ,2008(10).

[4] Yuexuan Wang, Junjie Chen, Yuping Huang etc, Research on Electromechanical Driving Technology and Development of Novel Rudder Servo Load Simulator[J], Aerospace control, 2014,32(2).

[5] Jeffrey travis, Jim kring, Labview for every: Graphical programming easy and fun [M], third edtion, 2006.

[6] Mathworks, “ Matlab Using Simulink”, The Math Works, Inc.2004. 